\title{
Téoros
}

Revue de recherche en tourisme

\section{La déréglementation du transport aérien aux États-Unis : «The sky is the limit.. "}

\section{Jean Pelletier}

Volume 9, numéro 2, juillet 1990

Le marché américain

URI : https://id.erudit.org/iderudit/1080032ar

DOI : https://doi.org/10.7202/1080032ar

Aller au sommaire du numéro

Éditeur(s)

Université du Québec à Montréal

ISSN

0712-8657 (imprimé)

1923-2705 (numérique)

Découvrir la revue

Citer cet article

Pelletier, J. (1990). La déréglementation du transport aérien aux États-Unis :

" The sky is the limit.. ». Téoros, 9(2), 43-46. https://doi.org/10.7202/1080032ar d'utilisation que vous pouvez consulter en ligne.

https://apropos.erudit.org/fr/usagers/politique-dutilisation/ 
L'observateur, aussiattentif soit-il, quitente de circonscrire le débat - vrai ou faux portant sur la déréglementation (et ses effets) dans le transport aérien américain, făit face à une série de préoccupations qui n'ont rien pour faciliter une compréhension claire des enjeux.

Que l'on se range derrière ceux qui défendent les impacts bénéfiques de la libéralisation ou que 1 'on prefere adopter un regard critique face à des situations difficilement contournables, force est de constater que le dossier n'est pas simple. Certains résultats escomptés dans les discours d'avant lamise en route de la déréglementation - c'était au milieu des années "70 - ne se sont pas matérialisés. En contrepartie, des problemes, soit ignorks, soit nouveaux, ont pris une ampleur considérable sans que des solutions adequates soient devenues des priorites.

Il suffirait de deux ou trois "erreurs humaines" successives dans des cabines de pilotage de gros porteurs américains ou dans des tours de contrôle des aéroports de New York ou de Chicago pour relancer la question de la nécessité ou pas d'un cadre réglementaire.

Nous écrivions en 1984 que "dans cinq ou dix ans, les partisans de la déréglementation comme ceux de mesures protectionnistes vont vraisemblablement continuer à faire valoir leurs points de vue respectifs au nom des consommateurs et des investisseurs"'(1). Il est évident que le vent continue à souffler derrière ceux qui favorisent la plus grande libéralisation possible et ce, à une échelle qui dépasse le continent nordaméricain. Â l'orée de l'Europe de '93 et avec la montée constante des forces économiques asiatiques, il semble clair que la globalisation des marchés a transformé les conditions nationales et internationales de la bataille du ciel... américain.

Jean Pelletier eat directeur géneral du Publicito club de Montrésl

\section{Rappel(s) et constats}

C'est en 1978 que le Congrès américain a adopté le Airlines Deregulation Act. La substitution des règles du marche aux mesures de contrôle gouvernemental s'est donc effectuée sous le règne d'un président democrate the oriquement beaucoup moins sensible que son successeur républicain aux avantages de la loi de l'offre et de la demande. Âl'époque, les consommateurs, les politiciens et les entreprises concernes par l'avenir du transport aérien se sont. entendus pour voir dans cette libéralisation convenue (complete mais progressivement réalisée en six ans) la source d'une concurrence accrue et, par voie de conséquence, une multiplication des transporteurs et une baisse généralisée destarifs, favorisant ainsi une accessibilité beaucoup plus grande à ce moyen modeme de déplacement pour nombre d'Américains.

Près de douze ans plustard, ondoit constater que la croissance du nombre de passagers transportés a été phénoménale: il s'agit d'uneaugmentationdeplus de $150 \%$ depuis 1978. Et, contre toute attente, on peut souligner, au cours de la même période, une diminution sensible du nombre ${ }^{*}$ accidents en relation au nombre beaucoup plus élevé d'heures de vol.

De toute évidence, le transport aérien constitue maintenant un mode populaire de déplacement qui ne cesse de croître malgré les problèmes observés (retards dû à l'engorgement des aéroports, baisse de la qualité du service, etc.).

Ce survol sommaire serait bien incomplet sans le constat que la part du marché des huit plus grosses compagnies qui était de $80 \%$ en 1978 est maintenant passée à ... 90\%. La concurrence (féroce comme nous le verrons plus loin) s'est traduite par une plus forte concentration. Étant donne la situation financière précaire de certains transporteurs, il n'est pas très risqué de penser qu'on se dirige allègrement (?) vers des situations quasimonopolistiques. Trois compagnies ont su profitersérieusement de la situation depuis 1978: American, Delta et United ont transporte $43 \%$ de tous les passagers qui ont voyage sur un avion américain en 1989.

Certains prétendent déjà que leur part de marché pourrait atteindre $50 \%$ avant la fin du siècle. Si des entreprises comme Trans World Airlines, Pan Am et Eastern volent toujours, c'est bientôt dans une chanson de Robert Charlebois que nous pourrons encore en suivre la trace. Il faut plutôt retenir les noms de Northwest, Continental et USAir comme compétiteurs crédibles au cours de la prochaine décennie au prix de montages financiers encore incertains, d'alliances difficilement prévisibles et de stratégies marketing probablement insoupçonnes.

Pour donner un aperçu des enjeux économiques, soulignons que pres de 500 millions de passagers ont été transportés aux USA en 1989: de façon conservatrice, les experts s'accordent à parler de 800 millions pour $1^{t}$ an 2000. American, Delta et United en ont respectivement transporté (en 1988) 64,58 et 56 millions $^{(2)}$. A titre d'exemple, la compagnie American qui, en 1989, a eu des recettes de 10,5 milliards \$ (U.S.) grâce à ses 544 appareils qui atterrissent dans 165 villes, a déja, comme ses principaux concurrents d'ailleurs, passé commande (ou pris des options) pour 544 appareils neufs de sorte qu'en 1997, 1'âge moyen de sa flotte soit passe de 9.6 ans a 8.4 ans $^{(5)}{ }_{+}$

La planification du contrôle du marché de la fin du siecle est une affaire d"actualité alors que le consommateur de maintenant est le "prisonnier" ou la victime heureuse ou malheureuse de la vive concurrence quotidienne à laquelle se livrentetles grands manitous de l'industrie et les petits futés qui réussissent parfois = du moins temporairement - à se bâtir une ou deux niches ${ }^{(4)}$.

Les megatransporteurs américains protegent quand même leurs arrières. Même sile 
numéroun American, en bonne santé financière grâce à un rythme de croissance annuel de l'ordre de $15 \%$, poursuit un ambitieux plan quinquennal de développement de plus de 20 milliards $\$$ (U.S.), il s'assure du contrôle de petites compagnies régionales susceptibles de lui fournir des clientèles locales à chaque escale de ses jets.

Nul n'est mieux servi que par soi-même: c'est le rôle des "commuters". En 1978, il existait 228 compagnies d'envergure régionale: la déréglementation aidant, leur nombre a chuté à près de 160 en 1990. Précisons que les 50 plus importantes contrôlent environ $95 \%$ du trafic. Etque 43 de ces 50 sont des filiales... les alimenteurs ("feeders"), affiliés à la compagnie American, et assurent quelques 1450 vols quotidiens pour rabattre des passagers à l'un ou l'autre des sept "hub" du transporteur ${ }^{(5)}$.

\section{Vive la déréglementation!}

Pour plusieurs des grands analystes américains, le changement de cap amorcé en 1978 s'avère positif. Qui plus est, un journal comme The New York Times, considéré généralement comme progressiste, vient, dans une série de deux éditoriaux, de prendre position: "Re-regulate? Not on Your Life ${ }^{+\hbar(6)}$. Le point de vue éditorial fait largement référence à une étude récente de deux chercheurs du Brookings Institution, étude qui tente de faire la démonstration du succès de la déréglementation en termes de baisses tarifaires et d'augmentation de la concurrence $^{()}$. Ce point de vue est contredit par une autre étude récente publiée cette fois par le Economic Policy Institute de Washingtonqui conclut de son côté a l'échec de la politique de la libéralisation à cause de ...l'augmentation des tarifs. Avec ces deux exemples qui amènent à une conclusion diamétralement opposée, on imagine facilement que les arguments ne s'appuient pas sur les mêmes prémisses ni sur les mêmes paramètres.

Il s'agit ici d'un débat quasi idéologique entre libéraux qui se sentent concernés par les problèmes socio-economiques (faillites, fusions, concentration) et environnementaux (congestions des aéroports, etc.) et économistes plus conservateurs quiépousent totalement les thèses traditionnelles de l'offre et de la demande. Voici unextrait du document de Messieurs Morrison et
Winston qui, de notre point de vue, témoigne d'une approche restrictive, mais combien significative permettant de conclure à la réussite du nouveau système:

"... While concentration is a concem, it is not a fair measure of competition among the airlines. The right measure is "route level competition": competition between carriers on specific routes. Based on our calculations, the number of effective competitors at the route level has risen from an average of 1.5 in 1978 to 1.9 in 1988 . This means that only 17 percent of passengers travel on carriers that control more than 90 percent of the traffic on a route. In 1978 , the figure was 28 percent. At the same time, 17 percent of passengers fly on carriers that control 20 percent or less of the traffic on their routes - up from 7 percent in 1978. Fewer carriers, yes, but they are competing with one another more often.

Our calculations also show that throughout the decade deregulated fares have been below regulated fares by an average of 18 percent. This amounts to average annual savings for travelers of roughly $\$ 6$ billion, calculated in 1988 dollars. But while fares have declined on average under deregulation, the spread of fares has increased. In 1978, less than half of 1 percent of travelers paid more than twice the average fare. In 1988, the figure was nearly 4 percent. In 1978 , only 10 percent of travelers paid fares less than 80 percent of the average. In 1988 , more than 25 percent did." $($ )

La double méthode de calcul est vraisemblablement rigoureuse mais permet de ne pas prendre en compte d'autres impacts de la concentration comme les dizaines et dizaines de mises à pied par suite de faillites et autres mesures dites de rationalisation. "Deregulation works. But only where is competition" concluait de son côté un éditorial paru dans le quotidien USA Today ${ }^{(8)}$. Comment assurer cette si nécessaire concurrence? Voici les suggestions de l'éditorialiste:
"- Airlines should not be allowed to use computer reservation systems to thwart competition.

- Airline mergers and buyouts that restrict competition should not be allowed.

- and laws against unfair competition and unfair practices can be strictly enforced."

A défaut de contrôler directement les tarifs et les permis des transporteurs, multiplions les autres mesures légaleset réglementaires. Après la fuite en avant, le retour gouvememental par les coulisses? Les médias parlent occasionnellement de la volonté de l'actuel secrétaire au transport (Samuel Skinner) du cabinet Bush de faire adopter éventuellement une "New national transportation Policy". Affaire à suivre.

Soulignons que si la concentration ne constitue pas àelle seule un critère suffisant pour mesurer le degré de concurrence, il convient d'être attentif à ce que les fusions et les acquisitions intervenant sur le marché déjà concentrés du transport aérien ne créent des positions de force sur le marché et n'accroissent ainsi le danger de collusion et de comportements anticoncurrentiels ${ }^{(9)}$.

Rappelons que durant le double mandat du président Reagan (1980-1988), aucun projet de fusion entre compagnies aériennes n'a été contesté par les responsables de Washington.

\section{La jungle aérienne}

La concurrence féroce des dix dernières années a fait nombre de victimes. Plusieurs se souviendront de l'entreprise vedette auprès des consommateurs quiétait devenu un objet d'études en milieu universitaire pour illustrer que le système américain n'avait pas son pareil pour permettre à de nouveaux joueurs d'occuper une place au soleil: son nom était Peoplexpress...

Dans tous les coins du pays, la loi de la jungle semble être la seule règle permise et ... admise. Deux exemples devraient illustrer cette recherche suicidaire d'une (meilleure) part du marché.

Trois millions de passagers ont volé en 1989 entre New York et Los Angeles à bord 
de vols directs (sans escale). C'est actuellement la deuxième route la plus achalandée dans le ciel américain. Jusqu'à récemment, cinq transporteurs (American, United, TWA, Pan Am et Continental) se livraient une lutte féroce (nombre de vols quotidiens, batailles tarifaires et mesures incitatives du genre abonnement "grands voyageurs") pour dominer ce marché majeur. Un sixième transporteur (MGM Grand), se contentant d'un modeste $1 \%$ de la clientèle, offre uniquement des sièges de 1 ère classe.

Mais voila qu'un septième transporteur (Delta) vient de lancer à son tour trois vols quotidiens (dans chaque direction). American a réagi en en ajoutant deux autres aux sept déja offerts. Continental et Pan Am entrent également dans la danse avec un vol additionnel chacune. Résultat: en moins de trois mois, le nombre de sièges disponibles a connu une croissance de $40 \%$... Quelle sera la première victime de cette lutte sans trêve et sans merci?

Et quiplus est, la bataille entre transporteurs américains est en trainde se répandre comme des traînées de poudre au-dessus de l'Atlantique et du Pacifique. Pendant des décennies, les porte-étandard aériens internationaux de la bannièrećtoilée étaient Pan Am et TWA au départ de New York, Chicago et Los Angeles ${ }^{(10)}$. Dorénavant, les liaisons entre les États-Unis et 1'Europe se multiplient: chaque grand transporteur, au départ de ses principales bases opérationnelles, cherche à développer des créneaux: c'est ainsi que USAir offre des vols entre Pittsburgh et Frankfort ou Delta entre Atlanta et Amsterdam. Le catalogue des nouvelles routes est à couper le souffle. On peut imaginer que les consommateurs profitent actuellement de ces offres nouvelles même si des destinations comme Glasgow ou Dusseldorf ont moinsd'attraits apparents que Londres et Paris.

La concurrence atlantique n'est cependant qu'une (importante) répétition de ce que le proche avenir va bientôt nous offrir audessus du Pacifique. Le développement du commerce avec les pays asiatiques prend une telle ampleur que le rythme annuel de croissance du nombre de passagers, qui se situe actuellement à $18 \%$, devrait passer à près de $25 \%$ d'ici quelques annees. Il n'est donc pas étonnant de constater que le Department of Transportation soit présentement saisi des demandes de douze transporteurs américains pour ouvrir de nouvelles routes aériennes à destination du Japon. Et il n'est pas surprenant de voir une compagnie comme Japan Airlines passer (mai 1990) une commande ferme de 20 Boeing 747-400 et prendre 34 options additionnelles sur ce quadriréacteur très long courrier ${ }^{(11)}$.

On assiste présentement en Extrême-Orient à une véritable explosion des déplacements due, d'une part àl'augmentation du pouvoir d'achat, d'autre part au développement rapide du commerce et de l'industrie et au succès du tourisme. Le village global, systèmes informatisés de réservations aidant, n'est pas loin.

\section{Pauvres passagers?}

L'expansion extraordinaire du trafic aérien constitue une indication certaine du succès de ce "nouveau mode de transport". Et toutes les prévisions assurent un avenir prometteur aux compagnies qui survivront.

Gens d'affaires et vacanciers vont continuer a être sollicités continuellement pour voyager souvent. La concurrence appelle des programmes pour fidéliser la clientèle d'affaires. Chez nos voisins du Sud, quelques 22 millions de voyageurs ont adhéré à un abonnement "grands voyageurs". L'accumulation du kilométrage effectué chez un même transporteur donne au voyageur le droit a une prime sous la formed'un voyage gratuit. Mis a part les $3 \%$ de voyageurs qui empruntent 1 'avion très régulièrement, rares sont les bénéficiaires qui réussissent soit à amasser suffisamment de points, soit a trouver le temps de profiter du voyage gratuit qui leur convient. II fautcomprendre que les offres sont très séduisantes mais les conditions d'exercice très contraignantes.

Quant au publicplusirégulier, enparticulier les vacanciers, il doit courir les aubaines et assumer la diversité des mesures restrictives comme l' achat 21 jours avant le départ d'un billet non remboursable. Les compagnies aériennes ont le gros bout du bâton. Grâce à des systèmes de réservations informatisés (SRI), elles détiennent une arme déterminante qui leur permet d'assigner un appareil (en terme de capacité) sur la meilleure route (en terme d'achalandage) et de pondérer la tarification en conséquence $^{(12)}$. Aux États-Unis, on enregistre des dizaines de milliers de changements tarifaires quotidiennement de sorte que les vols soient remplis même en périodes creuses. Il est facilede comprendre l'intérêt des transporteursd'être propriétaire de leur SRI et de les alimenter comme elles le veulent bien ${ }^{(13)}$.

Et, à moins d'avoir les moyens de voyager en classe affaires ou en première classe, il faut presqu'obligatoirement accepter de voyager assis dans un siège de plus en plus étroit et avec un espace pour les jambes de plus en plus restreint. La croissance du trafic est indéniable. Mais les conditions de voyages sont aussi indeniablement inadéquates. Peut-on espérer que la concentration des forces - les huit compagnies qui contrôlent environ $90 \%$ du marche - va maintenant encourager les transporteurs a se concurrencer au niveau du service? Rien n'est encore garanti. Les mêmes incertitudes demeurent en ce qui concerne la sécurité (terrorisme mis à part) et les aéroports. La croissance échevelée qui règne dans le transport aérien soulève les problemes aigus au sol comme dans les airs. A commencer par des problèmes de congestion aéroportuaires qui infligent aux usagers - une fois rendus au lieu de départ! - des retards croissants.

A l'heure actuelle, 21 des plus importants aéroports américains sont congestionnés. 20 autres le seront au fil des prochains dix ans. "You haven't seen anything yet" titrait Fortune récemment ${ }^{(14)}$. On estime à 44 milliards de dollars le coût des investissements nécessaires (nouvelles pistes, nouveaux systèmes de contrôle, nouveaux aéroports) pour faire face aux développements prévus. Pour l'instant (et pour longtemps), mieux vaut prévoir les retards même par beau temps.

Force est de constater cependant que le bilan de la sécurité aérienne dans le ciel américain est très satisfaisant depuis 1978 . Les plus âgés se souviendront que le président Reagan avait licencié 11400 des 16000 aiguilleurs du ciel qui avaient fait grève en 1981. Or, le nombre d'accidentset le tauxd'accidents fatalsn'ont pas augmenté jusqu'ici, bien au contraire. Il doit y avoir un dieu du ciel pour veiller au grain. Mếme si les instruments de vol dans les cabines de pilotage comme dans les tours de contrôle 
sont constamment perfectionnés, il n'en demeure pas moins qu' un tel résultat est quasi miraculeux.

Le cas de New York avec ses trois aéroports (Kennedy, La Guardia et Newark) est étonnant. En 1980,750 employés ont assuré les decollages et atterrissages d'un 1,1 million de vols. En 1989, quelque 530 aiguilleurs ont gére 1,74 million de vols: on parle ici d'environ 5200 départs et arrivées quotidiens. New York a reçu 75 millions de passagers en 1989; on parle de 115 millions en 2000. Il n'est donc pas surprenant de voir une ancienne base militaire maintenant ouverte aux vols commerciaux (Stewart Intemational Airport a $90 \mathrm{~km}$ au nord de Manhattan).

\section{Turbulences garanties}

Vu du Québec (où Air Alliance et Intair se livrent bataille) ou du Canada (où Air Canada et Canadien luttent pour protéger leur marché respectif), les enjeux du transport aérien américain semblent démesurés ${ }^{(15)}$.

A court terme, le transport aérien étant perçu comme une activité économique cyclique, il serait presomptueux de formuler des pronostics sur les résultats de chacun des transporteurs au cours des 36 prochains mois. Il serait déja moins hasardeux de prévoir que quelques entreprises vont changer de mains ou faire faillite. La concentration va progressivement se déplacer au plan international. Le phénomene de la globalisation est un fait qui se vérifie chaque jour et nous amène progressivement vers l'internationalisation des services.

On voit dejà nombre d'alliances intervenir entre transporteurs américains, européens et asiatiques (systemes de réservations, stratégiesmarketing etéchanges de capital). La nécessité de la présence de transporteurs américains dans le ciel de tous les continents va necessairement amener le gouvernement et, probablement, les consommateurs américains à se satisfaire d'une situation intérieure quasi monopolistique pour appuyer cette visibilité internationale. Seuls les excès véritablement abusifs - I'absence complete d"une "saine" concurrence sur une route spécifique - devraient faire l'objet de mesures antitrust.
Il ne faut pas perdre de vue également que les plus importants constructeursd'appareils (Boeing, McDonnell Douglas) sont américains et doivent se redéployer plus que jamais dans le secteur civil ${ }^{(16)}$. Cet impératif qui oblige les Américains à contribuer âture les principaux foumisseurs d'avions civils est un facteur additionnel susceptible de justifier nombre de pratiques commerciales a caractere monopolistiques $^{(17)}$.

Aux enjeux socio-cconomiques s'ajoutent donc des facteurs géopolitiques dont on ne doit pas minimiser l'importance. La nouvelle configuration mondiale (Europe 93, émergence des marchés asiatiques, libéralisation à l'Est) suggère (ou imposel) de regarder l'avenir avec intérêt et inquiétude. Les batailles sont clairement économiques sans camouflage paramilitaire. $f$

\section{NOTES}

(1) PELETIER, J, Une place au ciel pour tout te monde, Thoros, vol, 3, no 2, p. 20.

(2) Pour fin de comparaison, Air Canada et Canadien en ent respectivement transporté 11 et 9 millions (1988).

(9) SOURCE: SALOMON BROTHERS INC., USA TOday, 1er mai 1990, United et Delta ont pased des commandes de 474 at 354 appareils. Plus de 12 000 avions a resction seront livios aux compagnies abriennes mondiales au coura des 20 prochaines annees - un marché de 700 miliarda de dollara - aelon le conaortium eurcpeten Airbua qui vise à detenir quelque $30 \%$ de ce marcho face at Boeing et d quelques autres. Lo Monde, 10 mars 1990.

(4) La compagnie Midway aseure depuls quelques semaines des vola quotidenes entre Montréal et Philadelphie. Cette petite compagnie, qui rayonnait déjâ a pertir de Chicego, esseie de se doter d"un deuxieme moyeu ("hub") entre Washington, Battimere et un New York; la bataille sera rude.

(5) SALPUKAS, A., The Now York Timea, 18 mars 1990.

(6) 10 et 11 juin 1990 .

(7) MORIISON, SA. ET WINSTON, C., The Eeonomic Effects of Airline Dorogulatlon, 1990.

(8) Loteompetition Fix.Airlines Problems, 20 fevrier 1990 .

19 OCDE, Dereglementatlon et concurrence dans le transport adrien, Parię, 1988, pp. 78-92.

[10) En 1989, neut compagnies amb́ricaines ont transporté pras de 37 millions de peraonnes aur les routes intemationales (Canada compris). Pan Am et TWA réunies nien ont transportté que 13.6 millione.
(11) Màme une "petite" compegnie comme Kerean Airlines vient de passer aa commande de vingttrois 747-400 jpour b bagatelle somme de 4 mitlianda s U.S.).

(12) Aux Etato-Unis, on compte aur Apollo (United). SABRE [American] et Syatem one /Continental et Eastern). En Europe, ce sont Amadeus et Galiloo qui dominent. Dang un tel contexte, il n"est pas útonnant que les deux compagnies canadiennes -qui se livrent une rude concurrence-aient un SRI unifié.

(13) Il faut prendre acte que le ministère américain de la Justice manifeste quelques inquibtudes aur less effeta mencpolistiquea de la deréglementation puisqu'il mène préaentement une aérie d'enquätes pour comprendre les mócaniames de deciaione (reutes, tarifs, horaires, ete.) des compagnies.

(14) 18 juin 1990, pp. 104-110.

(15) Le transport transfrontalier canado-amoricain fait I'objet d'un acoerd bilatéral nogecis on 1966 qui n'a pasa été pris en compte lora des tractationa qui ont mené à l'Aocord de libre-6change.

(16) On peut présumer qu'il en va de même pour les fabricants aovistiques. Mais qui a voyage aur les avione d'Abroflot ne stattend pas di une forte oompotition do lour part an matiore de renouvellement des flottes nationales. Le coneortium Aibua pour sa part peut prótendro a quelque $30 \%$ du marche des future appeareils.

(17) Lacempagnie Boeing a un carnet de commandes de 1750 avions $(737,747,757$ et 767$)$ sans compter l'Airbus. A330 et de remplacer lea Tristar ot DCB vieilianants. 